\title{
Ketoconazole: Solving the Poor Solubility via Cocrystal Formation with Phenolic Acids
}

Xin Chen, Duanxiu Li, Zongwu Deng and Hailu Zhang*

Laboratory of Magnetic Resonance Spectroscopy and Imaging, Suzhou Institute of Nano-Tech and Nano-Bionics, Chinese Academy of Sciences, Suzhou 215123, P. R. China.

*Corresponding Author. E-mail: hlzhang2008@sinano.ac.cn; Tel: +86-512-62872713; Fax: $+86-512-62603079$. 


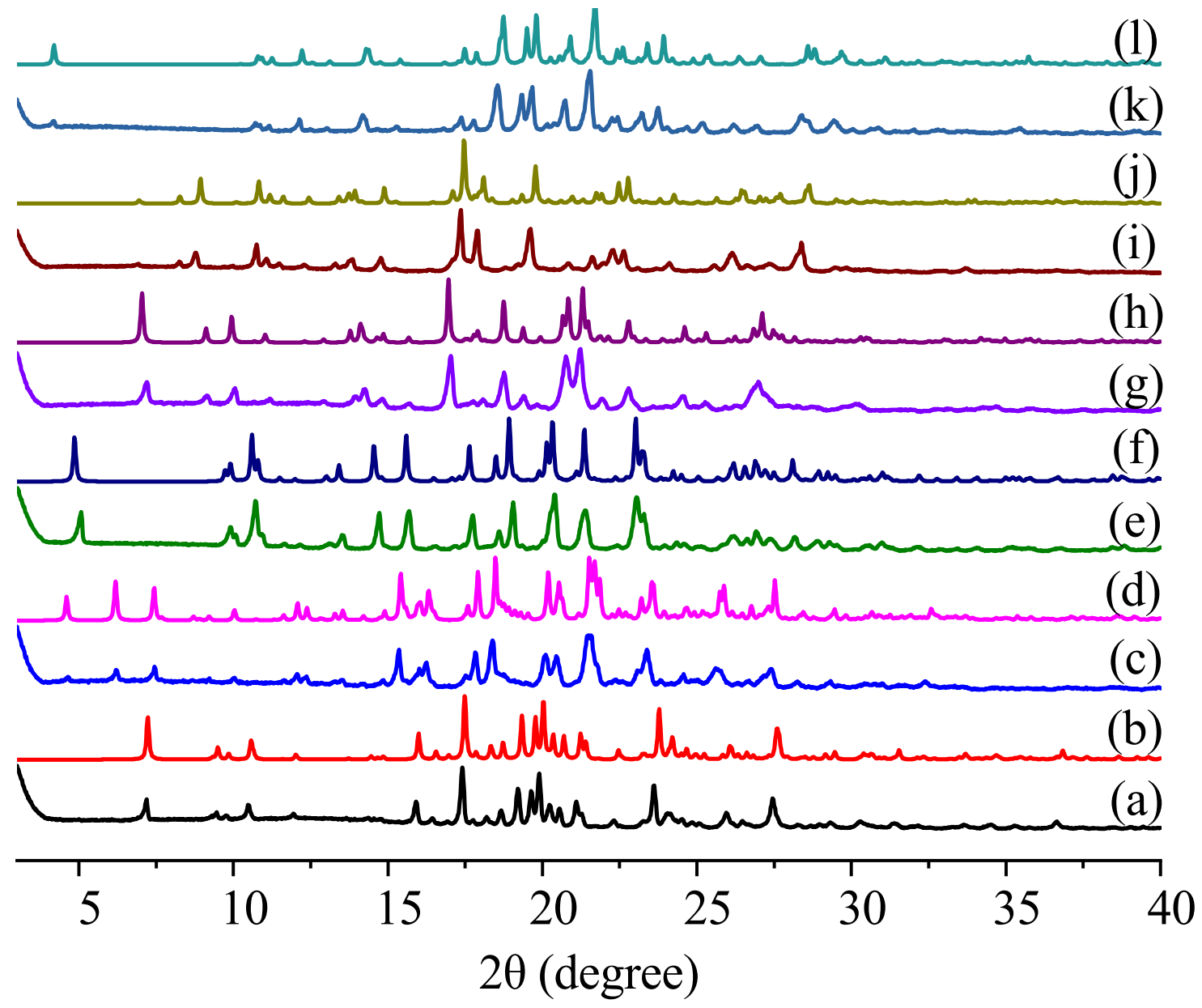

Figure S1 Experimental and simulated powder XRD patterns of KTZ (a and b), KTZ24DHB (c and d), KTZ-25DHB (e and f), KTZ-34DHB (g and h), KTZ-GA (i and j), and KTZ-TPCA (k and 1). 


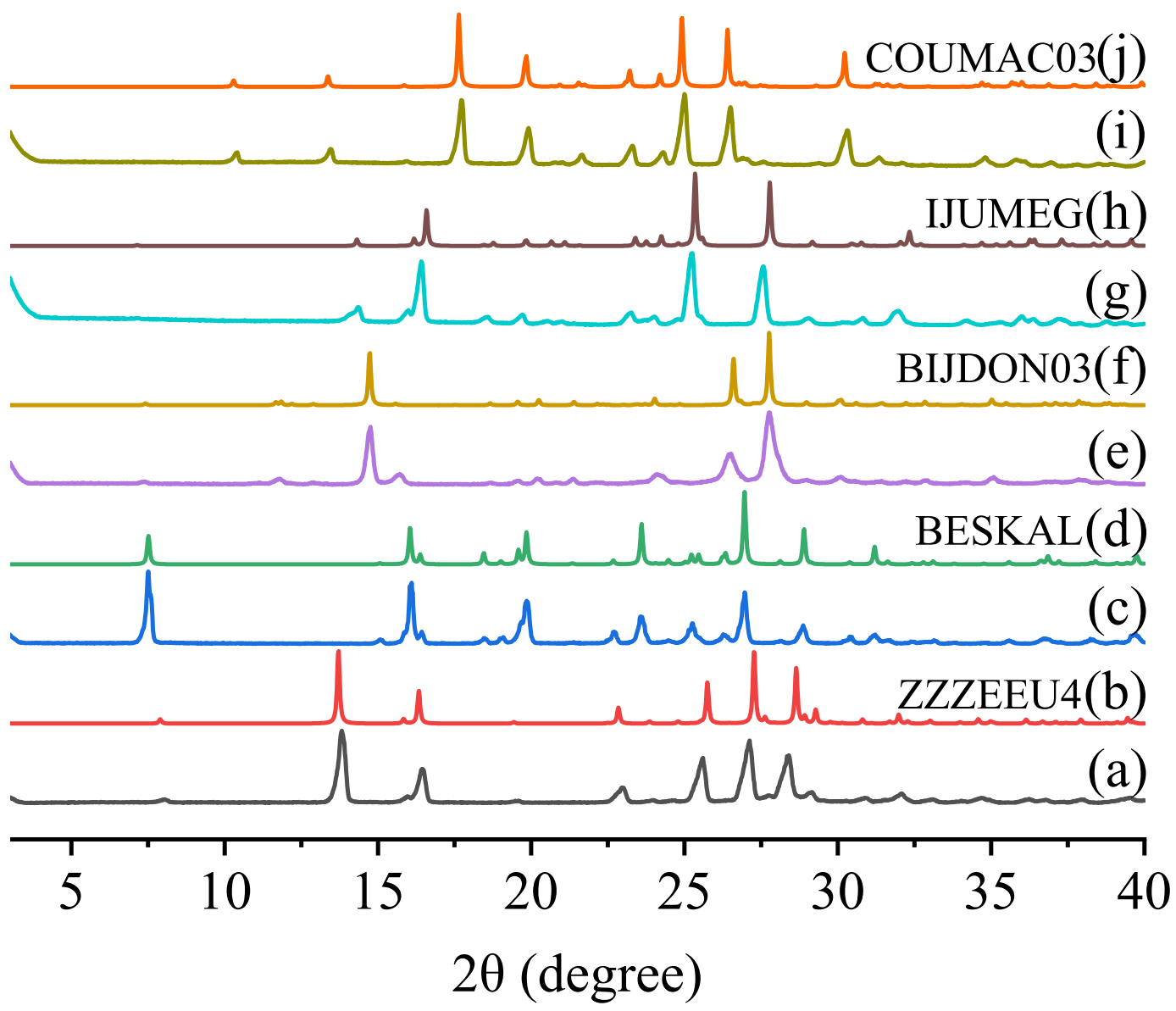

Figure S2 Experimental and simulated powder XRD patterns of 24DHB (a and b), 25DHB (c and d), 34DHB (e and f), GA (g and h), and TPCA (i and j). The CSD refcodes of the used structures are provided along with the simulated patterns. 
(f)

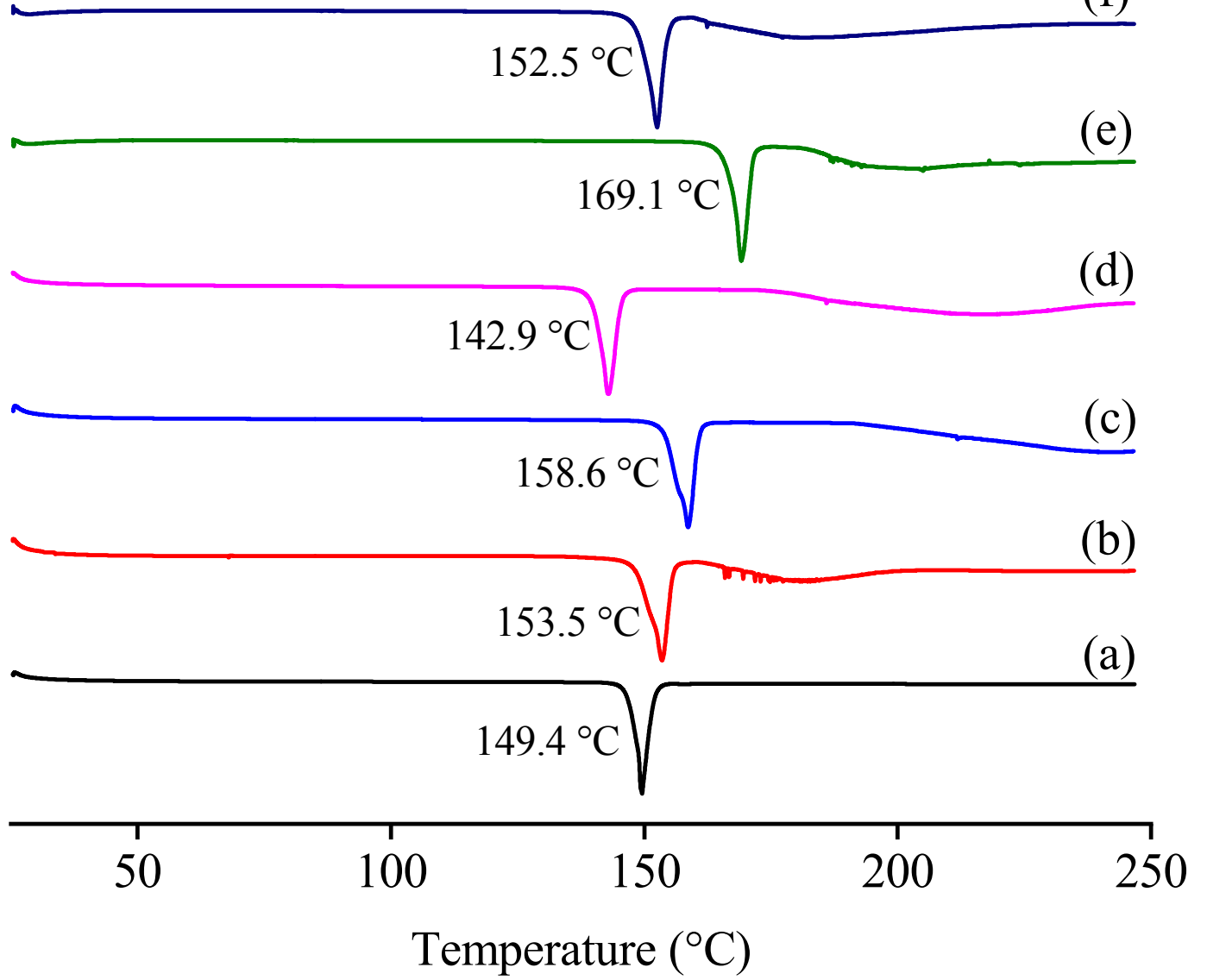

Figure S3 DSC curves of KTZ (a), KTZ-24DHB (b), KTZ-25DHB (c), KTZ-34DHB (d), KTZ-GA (e), and KTZ-TPCA (f). 


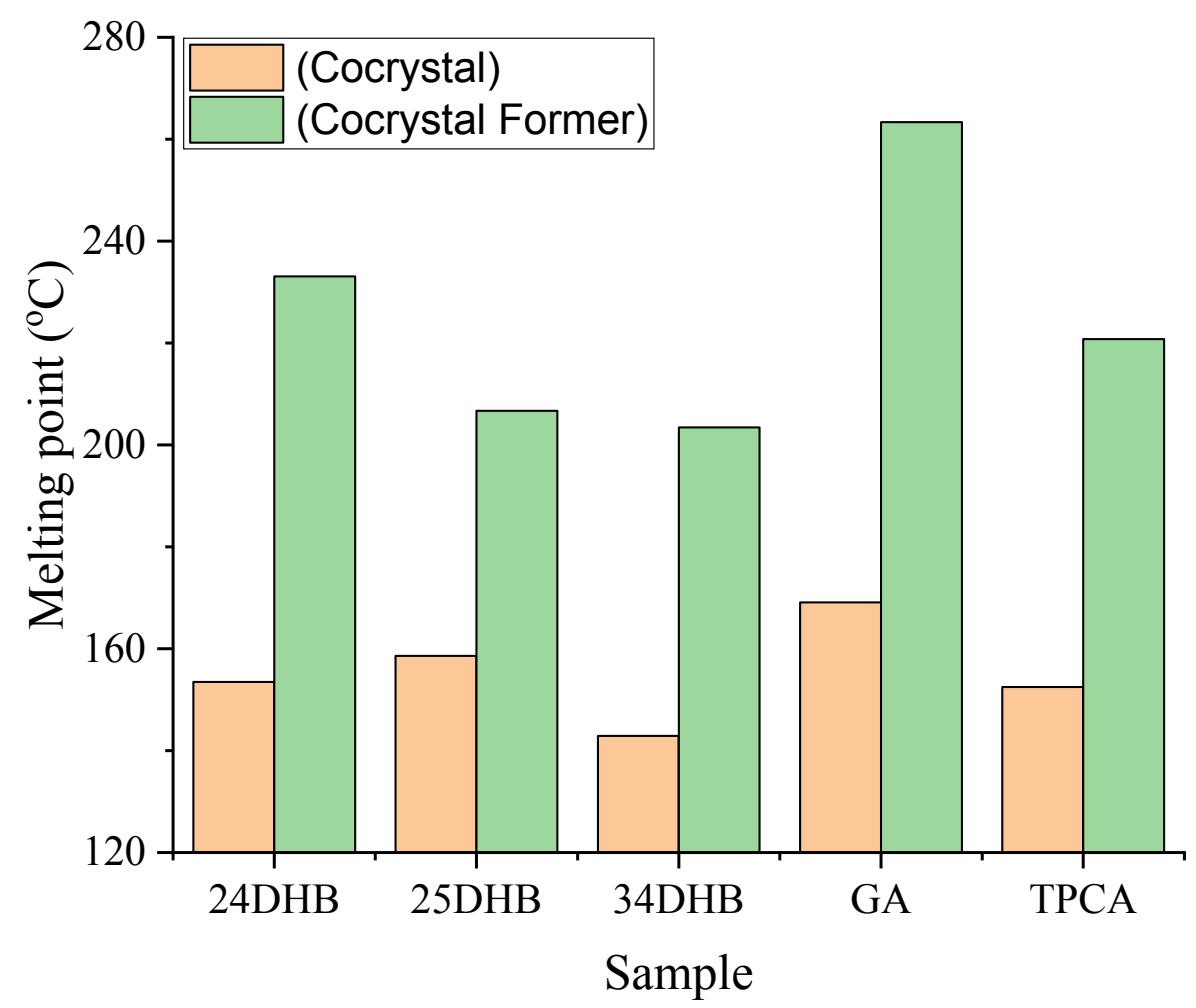

Figure S4 Melting points ( $T_{\max }$ ) of KTZ cocrystals and the cocrystal formers. 


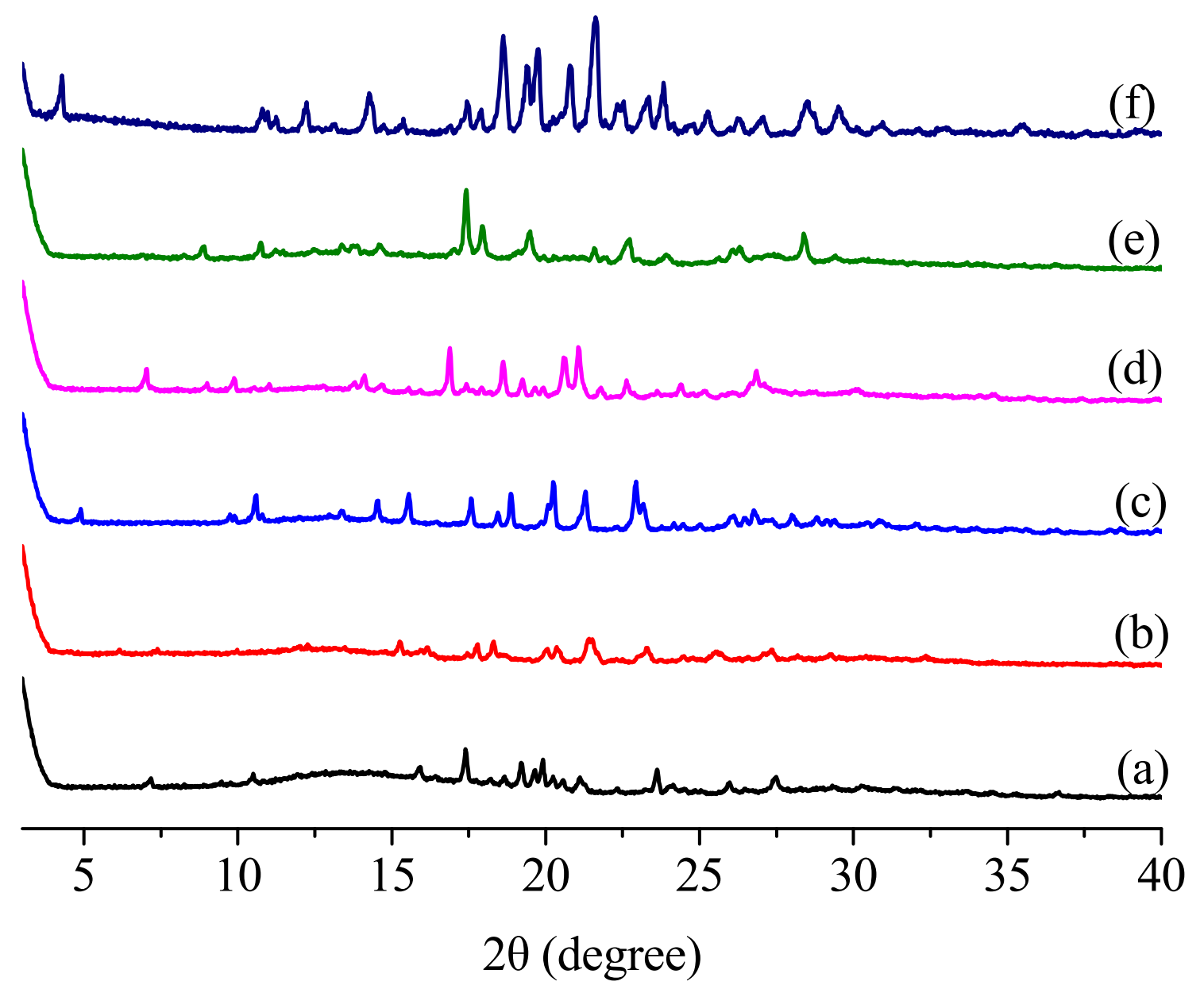

Figure S5 Powder XRD patterns of KTZ (a), KTZ-24DHB (b), KTZ-25DHB (c), KTZ34DHB (d), KTZ-GA (e), and KTZ-TPCA (f) after dissolution experiment in ultrapure water. 
Table S1. Hydrogen bonding distances and angles of KTZ cocrystals.

\begin{tabular}{|c|c|c|c|c|}
\hline $\mathrm{D}-\mathrm{H} \cdots \mathrm{A}$ & $\mathrm{H} \cdots \mathrm{A}(\AA)$ & $\mathrm{D} \cdots \mathrm{A}(\AA)$ & $\mathrm{D}-\mathrm{H} \cdots \mathrm{A}(\mathrm{deg})$ & Symmetry codes \\
\hline \multicolumn{5}{|c|}{ KTZ-24DHB } \\
\hline $\mathrm{O} 5 \mathrm{~A}-\mathrm{H} 5 \mathrm{C} \cdots \mathrm{N} 4 \mathrm{~A}$ & $1.43(8)$ & $2.528(5)$ & $167(8)$ & \\
\hline $\mathrm{O} 8 \mathrm{~A}-\mathrm{H} 8 \mathrm{AA} \cdots \mathrm{O} 1$ & $1.75(5)$ & $2.601(4)$ & $177(5)$ & $\mathrm{x}, \mathrm{y},-1+\mathrm{z}$ \\
\hline $\mathrm{O} 5-\mathrm{H} 5 \cdots \mathrm{N} 4$ & $1.73(6)$ & $2.580(5)$ & $176(6)$ & $\mathrm{x}, \mathrm{y},-1+\mathrm{z}$ \\
\hline $\mathrm{O} 7-\mathrm{H} 7 \mathrm{~A} \cdots \mathrm{O} 6$ & $1.86(8)$ & $2.591(4)$ & $142(7)$ & \\
\hline O7A-H7AA $\cdots$ O6A & $1.67(4)$ & $2.567(5)$ & $148(4)$ & \\
\hline $\mathrm{O} 8-\mathrm{H} 8 \mathrm{~B} \cdots \mathrm{O} 1 \mathrm{~A}$ & $1.70(6)$ & $2.646(4)$ & $169(4)$ & \\
\hline $\mathrm{C} 14-\mathrm{H} 14 \cdots \mathrm{N} 2 \mathrm{~A}$ & 2.48 & $3.464(4)$ & 171 & \\
\hline $\mathrm{C} 23 \mathrm{~A}-\mathrm{H} 23 \mathrm{C} \cdots \mathrm{O} 6$ & 2.35 & $3.298(5)$ & 163 & $\mathrm{x}, 1+\mathrm{y}, 1+\mathrm{z}$ \\
\hline $\mathrm{C} 25 \mathrm{~A}-\mathrm{H} 25 \mathrm{~A} \cdots \mathrm{N} 2$ & 2.56 & $3.369(5)$ & 144 & $2-\mathrm{x}, 1-\mathrm{y}, 2-\mathrm{z}$ \\
\hline $\mathrm{C} 26 \mathrm{~A}-\mathrm{H} 26 \mathrm{~A} \cdots \mathrm{N} 4$ & 2.59 & $3.394(5)$ & 143 & $\mathrm{x}, 1+\mathrm{y}, \mathrm{z}$ \\
\hline \multicolumn{5}{|c|}{ KTZ-25DHB } \\
\hline $\mathrm{O} 5-\mathrm{H} 5 \cdots \mathrm{N} 4$ & $1.55(4)$ & $2.546(3)$ & $166(4)$ & \\
\hline $\mathrm{O} 7-\mathrm{H} 7 \cdots \mathrm{O} 6$ & $1.79(4)$ & $2.591(3)$ & $154(4)$ & \\
\hline $\mathrm{O} 8-\mathrm{H} 8 \mathrm{~A} \cdots \mathrm{O} 1$ & $1.83(3)$ & $2.661(3)$ & $174(3)$ & $\mathrm{x}, 1 / 2-\mathrm{y}, 1 / 2+\mathrm{z}$ \\
\hline $\mathrm{C} 24-\mathrm{H} 24 \cdots \mathrm{O} 6$ & 2.30 & $3.192(3)$ & 160 & $1-x,-1 / 2+y, 3 / 2-z$ \\
\hline $\mathrm{C} 31-\mathrm{H} 31 \cdots \mathrm{O} 8$ & 2.55 & $3.378(4)$ & 148 & $-\mathrm{x}, 1-\mathrm{y}, 1-\mathrm{z}$ \\
\hline \multicolumn{5}{|c|}{ KTZ-34DHB } \\
\hline $\mathrm{O} 5-\mathrm{H} 5 \cdots \mathrm{O} 1$ & $1.82(3)$ & $2.614(3)$ & $161(3)$ & $\mathrm{x}, \mathrm{y}, 1+\mathrm{z}$ \\
\hline $\mathrm{O} 7-\mathrm{H} 7 \cdots \mathrm{O} 8$ & $1.87(4)$ & $2.720(3)$ & $142(3)$ & $-\mathrm{x}, 1-\mathrm{y},-\mathrm{z}$ \\
\hline $\mathrm{O} 8-\mathrm{H} 8 \mathrm{~B} \cdots \mathrm{N} 4$ & $1.87(3)$ & $2.678(3)$ & $172(3)$ & $-\mathrm{x}, 1-\mathrm{y},-\mathrm{z}$ \\
\hline $\mathrm{C} 15-\mathrm{H} 15 \mathrm{~A} \cdots \mathrm{O} 4$ & 2.51 & $3.316(6)$ & 140 & $1-\mathrm{x},-\mathrm{y},-\mathrm{z}$ \\
\hline $\mathrm{C} 23-\mathrm{H} 23 \mathrm{~A} \cdots \mathrm{O} 1$ & 2.34 & $3.258(3)$ & 158 & $\mathrm{x},-1+\mathrm{y}, 1+\mathrm{z}$ \\
\hline \multicolumn{5}{|c|}{ KTZ-GA } \\
\hline $\mathrm{O} 5-\mathrm{H} 5 \cdots \mathrm{O} 6$ & $1.72(4)$ & $2.606(3)$ & $178(5)$ & $1-\mathrm{x}, 2-\mathrm{y},-\mathrm{z}$ \\
\hline $\mathrm{O} 7-\mathrm{H} 7 \cdots \mathrm{N} 4$ & $1.83(4)$ & $2.647(3)$ & $166(4)$ & \\
\hline $\mathrm{O} 8-\mathrm{H} 8 \mathrm{~A} \cdots \mathrm{O} 7$ & $2.04(4)$ & $2.745(3)$ & $145(4)$ & $1-\mathrm{x}, 1-\mathrm{y}, 1-\mathrm{z}$ \\
\hline $\mathrm{O} 9-\mathrm{H} 9 \mathrm{~A} \cdots \mathrm{O} 1$ & $1.78(4)$ & $2.635(4)$ & $177(5)$ & $-\mathrm{x}, 2-\mathrm{y}, 1-\mathrm{z}$ \\
\hline $\mathrm{C} 3-\mathrm{H} 3 \mathrm{~B} \cdots \mathrm{O} 1$ & 2.60 & $3.458(8)$ & 148 & $-\mathrm{x}, 2-\mathrm{y}, 1-\mathrm{z}$ \\
\hline $\mathrm{C} 15-\mathrm{H} 15 \mathrm{~A} \cdots \mathrm{O} 6$ & 2.54 & $3.505(5)$ & 176 & $1-\mathrm{x}, 1-\mathrm{y},-\mathrm{z}$ \\
\hline $\mathrm{C} 26-\mathrm{H} 26 \cdots \mathrm{O} 8$ & 2.47 & $3.078(4)$ & 123 & $1-\mathrm{x}, 1-\mathrm{y}, 1-\mathrm{z}$ \\
\hline \multicolumn{5}{|c|}{ KTZ-TPCA } \\
\hline
\end{tabular}




\begin{tabular}{|c|c|c|c|c|}
\hline $\mathrm{O} 5-\mathrm{H} 5 \cdots \mathrm{N} 4$ & $1.70(4)$ & $2.635(3)$ & $178(4)$ & \\
\hline $\mathrm{O} 7-\mathrm{H} 7 \cdots \mathrm{O} 1$ & $1.93(3)$ & $2.758(3)$ & $170(4)$ & $-\mathrm{x}, 1 / 2+\mathrm{y}, 3 / 2-\mathrm{z}$ \\
\hline $\mathrm{C} 9-\mathrm{H} 9 \cdots \mathrm{O} 6$ & 2.48 & $3.236(3)$ & 138 & $\mathrm{x}, 3 / 2-\mathrm{y},-1 / 2+\mathrm{z}$ \\
\hline $\mathrm{C} 15-\mathrm{H} 15 \mathrm{~A} \cdots \mathrm{O} 5$ & 2.54 & $3.407(4)$ & 148 & $1-\mathrm{x}, 2-\mathrm{y}, 1-\mathrm{z}$ \\
\hline $\mathrm{C} 23-\mathrm{H} 23 \mathrm{~B} \cdots \mathrm{O} 6$ & 2.54 & $3.349(3)$ & 141 & $1-x, 1 / 2+y, 3 / 2-z$ \\
\hline
\end{tabular}


Table S2. The $\mathrm{pH}$ values after dissolution experiments.

\begin{tabular}{ccccccc}
\hline Sample & KTZ & $\begin{array}{r}\text { KTZ- } \\
\text { 24DHB }\end{array}$ & $\begin{array}{r}\text { KTZ- } \\
\text { 25DHB }\end{array}$ & $\begin{array}{c}\text { KTZ- } \\
\text { 34DHB }\end{array}$ & KTZ-GA & $\begin{array}{c}\text { KTZ- } \\
\text { TPCA }\end{array}$ \\
\hline $\mathrm{pH}$ & 7.44 & 5.35 & 4.92 & 4.70 & 4.46 & 5.86 \\
\hline
\end{tabular}

